\title{
INTERFACE, GUIDED AND NON-LOCALIZED MODES IN SUPERLATTICES
}

\author{
R. Brazis, R. NARKoWICZ \\ Semiconductor Physics Institute, Gostauto 11, 2600 Vilnius, Lithuania \\ and \\ Polish University at the Association of Polish Scientists of Lithuania \\ P.O. Box 823, 2055 Vilnius, Lithuania. \\ AND L. SaFonova \\ Semiconductor Physics Institute, Gostauto 11, 2600 Vilnius, Lithuania
}

The numerical dispersion calculations in long-period GaAs/AlAs superlattice were performed using a local theory with retardation as well as an effective medium approximation. The splitting of the dispersion branches near the transverse optical phonon frequencies and phonon-like to photon-like modes transition were found to depend on the superlattice spatial period. The characteristic frequencies of bulk modes and crossing point of dispersion branches estimated analytically with the use of an effective medium approximation were found to agree with the results of rigorous solution.

PACS numbers: 78.66.-w

\section{Introduction}

Semiconductor superlattices are known to support the variety of modes either non-localized or localized within the layers or at the interfaces. In a long-wavelength limit the superlattice may be considered as a homogeneous uniaxial medium with a tensorial dielectric permeability $[1,2]$. This effective medium approximation yields the non-localized (bulk) modes arising from the correlated polarization of the superlattice as a whole. The effective dielectric tensor is intensively used to describe experimental results $[3,4]$. Much less work has been done in the field of bulk modes dispersion, transformation into other modes or coexistence with them. The aim of present work is to study various modes dispersion in superlattice in order to estimate their existence conditions as well as the place in the entire excitation spectrum. In-plane propagation case was chosen as the most convenient for this purpose. 


\section{Theory}

Theoretical investigation is based on a local electromagnetic theory, which is known to be valid for layer thickness values above $100 \AA$. The electromagnetic field in particular layers obeys the Maxwell equations with boundary conditions of continuity and periodicity of the tangential components at the interfaces. The layers are characterized by macroscopic frequency-dependent dielectric functions of corresponding bulk materials

$$
\varepsilon_{j}(\omega)=\left(\varepsilon_{\infty}\right)_{j} \frac{\omega^{2}-\omega_{\mathrm{L} j}^{2}}{\omega^{2}-\omega_{T j}^{2}}
$$

where $\varepsilon_{\infty}$ is the high-frequency dielectric constant, $\omega_{\mathrm{L}}$ and $\omega_{\mathrm{T}}$ are the frequencies of LO- and TO-phonons respectively, $j=1,2$ is the index of the layer. The effects of free carriers as well as of intrinsic phonon damping are neglected. Layer interfaces are assumed to be perfectly smooth. With these assumptions the dispersion equation of an infinite binary superlattice for $p$-polarized wave may be written as follows:

$$
\cos \alpha_{1} d_{1} \cos \alpha_{2} d_{2}-\frac{\varepsilon_{1}^{2} \alpha_{2}^{2}+\varepsilon_{2}^{2} \alpha_{1}^{2}}{2 \varepsilon_{1} \varepsilon_{2} \alpha_{2} \alpha_{2}} \sin \alpha_{1} d_{1} \sin \alpha_{2} d_{2}=\cos k_{\perp} d
$$

where $\alpha_{1,2}=\left[k_{0}^{2} \varepsilon_{1,2}(\omega)-k_{\|}^{2}\right]^{1 / 2}, d_{1,2}$ are the thicknesses of the layers, $k_{\|, \perp}$ are respectively the in-plane and transverse wave vectors, $k_{0}=\omega / c, d=d_{1}+d_{2}$ is the period of the superlattice.

For the case of in-plane propagation $\left(k_{\perp}=0\right)$ in the limit of $\alpha_{j} d_{j} \ll 1$ we obtained two dispersion equations in the effective medium approximation

$$
\begin{aligned}
& k_{\|}^{2}=k_{0}^{2} \varepsilon_{\|}=k_{0}^{2} \frac{\varepsilon_{1} \varepsilon_{2} d}{\varepsilon_{1} d_{2}+\varepsilon_{2} d_{1}}, \\
& \varepsilon_{1} d_{1}=-\varepsilon_{2} d_{2} .
\end{aligned}
$$

The former of them gives the in-plane propagation equation where $\varepsilon_{\|}$is the longitudinal component of superlattice dielectric tensor, whereas the latter describes the set of straight lines $\omega=$ const.

\section{Results and discussion}

The numerical solution of Eq. (1) was performed for GaAs/AlAs superlattices with two values of period but the same layer thickness ratio. Indices 1 and 2 are taken for GaAs and AlAs respectively.

The dispersion picture near the restrahlen regions of the superlattice materials is presented in Fig. 1 together with the results in effective medium approximation. The poles of $\varepsilon_{\|}$can be estimated from Eq. (3.1):

where

$$
\omega_{\mathrm{b}}^{ \pm}=\frac{P_{\mathrm{b}} \pm\left[P_{\mathrm{b}}-4 Q_{\mathrm{b}}(1+l / r)\right]^{1 / 2}}{2(1+l / r)}
$$

$$
P_{\mathrm{b}}=\omega_{\mathrm{L} 1}^{2}+\omega_{\mathrm{T} 2}^{2}+(l / r)\left(\omega_{\mathrm{L} 2}^{2}+\omega_{\mathrm{T} 1}^{2}\right),
$$




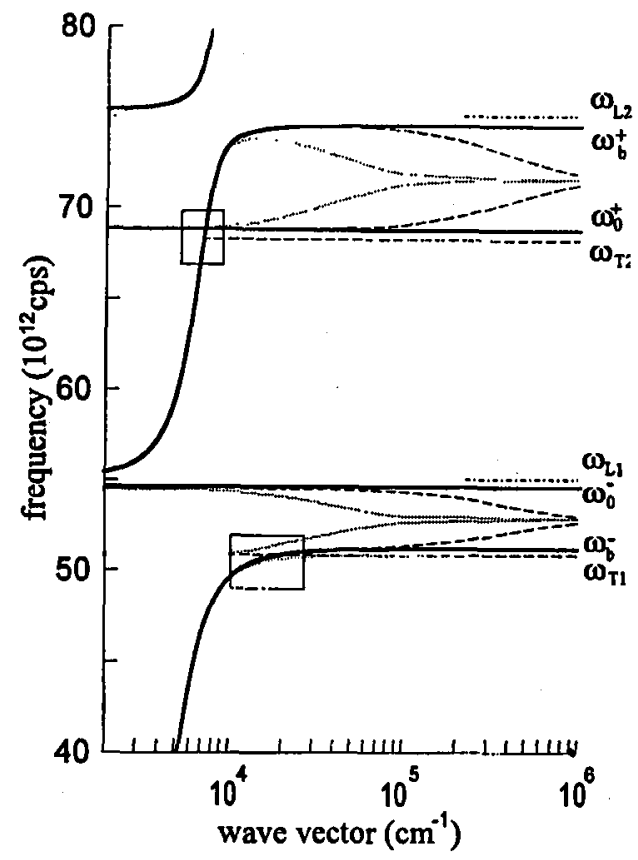

Fig. 1. The dispersion branches in GaAs/AlAs superlattice with layer thickness ratio $d_{1} / d_{2}=9 . d=2.5 \mu \mathrm{m}$ for the dotted lines and $d=0.25 \mu \mathrm{m}$ for the dashed ones. Solid curves represent the EM approximation.

$$
\begin{aligned}
& Q_{\mathrm{b}}=\omega_{\mathrm{L} 1}^{2} \omega_{\mathrm{T} 2}^{2}+(l / r)\left(\omega_{\mathrm{L} 2}^{2} \omega_{\mathrm{T} 1}^{2}\right), \\
& l=\left(\varepsilon_{\infty}\right)_{2} /\left(\varepsilon_{\infty}\right)_{1}, \quad r=d_{2} / d_{1} .
\end{aligned}
$$

Equation (3.1) gives also two zeros of $\varepsilon_{\|}$(i.e. $\omega_{L j}$ ). The cut-off frequencies of non-dispersive branches $\omega_{0}^{ \pm}$, described by Eq. (3.2), can be obtained by substituting $r$ instead of $1 / r$ in Eq. (4).

One can suggest non-localized modes to exist in the regions of the coincidence with effective medium (EM) approximation. This coincidence seems to be very good outside the reststrahlen bands (solid curves in Fig. 1). The bulk-like transverse excitations do exist there with the field distributed nearly uniformly in the unit cell of superlattice (SL) (the lowest solid curve in Fig. 1), or concentrated in the particular kind of layers (two upper solid curves in Fig. 1). The guiding layers are these with $\omega_{\mathrm{L}}$ giving the origin to the corresponding branches [5].

Two kinds of non-localized modes were found to exist inside the restrahlen regions. One of them corresponds to the maximum on the dispersion branch at the resonant frequency $\omega_{\mathrm{b}}^{+}$(Fig. 1) or $\omega_{\mathrm{b}}^{-}$(if $d_{1}<d_{2}$ ). Another kind of non-localized modes are found in the restrahlen regions of both materials at the frequencies $\omega \approx \omega_{0}^{ \pm}$and wave vectors $k_{\|}<1 / d$. These are nondispersive phonon-like modes.

Bulk-to-interface mode transition is clearly seen in Fig. 1 at $k_{\|}>1 / d$. It gives rise to positive or negative dispersion in restrahlen regions provided that $d_{1} \neq d_{2}$. 

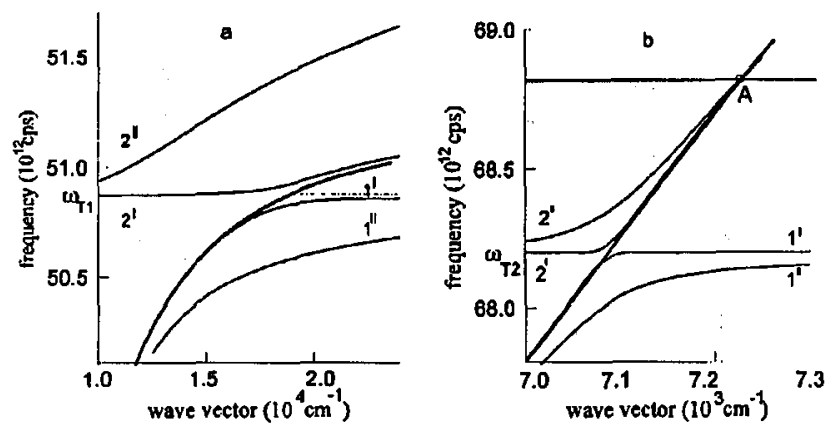

Fig. 2. The dispersion picture near TO-phonon frequency of the thicker (a) and the thinner (b) layers of superlattice, corresponding to the marked regions in Fig. 1. $d=0.25 \mu \mathrm{m}\left(1^{\prime}, 1^{\prime \prime}\right), d=2.5 \mu \mathrm{m}\left(2^{\prime}, 2^{\prime \prime}\right)$.

Let us confine our attention to the regions near $\omega_{\mathrm{T} j}$ (marked regions in Fig. 1). It should be noted that the higher-order modes situated in this region [5] are out of scope of the present work. It is seen in Fig. 2a,b that none of dispersion branches crosses $\omega_{\mathbf{T} j}$, as it is predicted by EM approximation. The modes in this regions correspond to the mixed phonon-like and photon-like excitations. The dispersion branches below $\omega_{\mathrm{T} j}$ tending to this frequency with the rise of $k_{\|}$ show the transition from the bulk or slab-guided modes $\left(1^{\prime}, 1^{\prime \prime}\right.$. in Fig. $2 a$ and $b$, respectively) to the confined TO-modes. Dispersion above $\omega_{\mathrm{T} j}$ demonstrates the transition to the bulk (or interface) modes. If $j$ is an index of the thinner layer (Fig. 2b), the branch $2^{\prime}\left(2^{\prime \prime}\right)$ is that one possessing a maximum. In the case of the thicker layer (Fig. 2a) the corresponding branch represents rather virtual bulk mode influenced strongly by TO-phonons. The upper straight line demonstrates an excellent coincidence of phonon-like mode dispersion with EM approximation results. The position of the branch crossing point $A$ can be obtained from Eqs. (3.1) and (3.2):

$$
k_{\|}^{2}(A)=k_{0}^{2} \frac{\left(\varepsilon_{\infty}\right)_{i} d_{i}\left[\omega^{2}(A)-\omega_{L i}^{2}\right]}{\left(d_{i}-d_{k}\right)\left[\omega^{2}(A)-\omega_{T i}^{2}\right]}, \quad \omega(A)=\omega_{0}^{ \pm}(A),
$$

where $i$ and $k$ are the indexes of the thinner and the thicker layer respectively, the sign " + " in $\omega_{0}$ is taken if $\omega_{T i}>\omega_{T k}$ and "-" is taken in the opposite case. It can be shown that $k_{\|}(A)$ and $\omega(A)$ give the trivial solution of Eq. (1). Therefore in the absence of damping the branch crossing point is an inherent feature of dispersion in SL with non-equal layer thicknesses $\left(d_{1} \neq d_{2}\right)$.

\section{References}

[1] N. Raj, D.R. Tilley, Solid State Commun. 55, 373 (1985).

[2] V.M. Agranovich, V.E. Kravtsov, Solid State Commun. 55, 85 (1985).

[3] M. Haraguchi, M. Fukui, S. Muto, Phys Rev. B 41, 1254 (1990).

[4] T. Dumelow, A.A. Hamilton, T.J. Parker, D.R. Tilley, C.T.B. Foxon, D. Hilton, K.J. Moore, Int. J. Infrared Millim. Waves 11, 901 (1990).

[5] R. Brazis, L. Safonova, R. Narkowicz, Infrared Phys. Technol., to be published. 